proving the animal's condition, actually precipitated respiratory arrest.

C. A. de Candolie

M. K. MePhatL

Suffield Experimental Station,

Ralston, Alberta,

Canada. April 28. ${ }^{1}$ Paton, W. D. M., and Perry, W. L. M., J. Physiol., 112, 48 P (1951);
119, 43 (1953).

${ }^{2}$ Douglas, W. W., and Matthews, P. B. C., J. Physiol., 116, 202 (1952).

\section{Excretion in Human Urine of an Unknown Amino-acid derived from Dates}

$I_{N}$ the course of an investigation with the aid of paper chromatography of the genetics of amino-acid excretion in man, it was observed that some individuals excreted large quantities of an unknown ninhydrin-positive substance. Upon inquiry concerning the diet of these individuals, it was found that dates had been eaten in all cases. Seven individuals were selected who had not eaten dates for at least a month, and chromatograms of their urine were prepared. In no case was the 'date spot' observed. 'These seven individuals were then fed eight to ten dates in the evening, and the following morning a sample of their urine was collected. Another urine sample was obtained the following morning. In every case the chromatograms of the first urine specimen showed quite clearly the cdate spot'. The chromatograms of the urine specimens produced on the second day after eating dates showed only a trace of this spot, or failed to show it altogether.

The unknown substance which produces the 'date spot' moves on the two-dimensional chromatograms in phenol-lutidine solvents to a location almost identical with that of $\alpha$-amino- $n$-butyric acid. However, in butanol-acetic $(4: 1: 1)$ solvent, this substance moves much less than $\alpha$-amino- $n$-butyric acid, its $R_{F}$ being about $0 \cdot 25$. The colour of the spot after developing with ninhydrin and heating also differentiates it from $\alpha$-amino- $n$-butyric acid. The 'date spot' is clear blue, resembling somewhat the colour given on similar chromatograms by aspartic acid, rather than the violet or purple given by most other amino-acids. This blue becomes gradually more purple after standing for several days.

We have also investigated the occurrence of this substance in the date, and find that it is. present in the free form and does not appear to increase in amount after acid hydrolysis. This suggests that the substance is an amino-acid and not a peptide. A copper complex test has also been run indicating that the substance is an $\alpha$-amino-acid.

It is interesting that the amino-acid under consideration is excreted apparently unchanged, since from superficial appearances its excretion appears to be quantitative. This is a rather unusual situation for amino-acid metabolism in the human body; but we have no positive proof that the excretion is strictly quantitative.

This work was carried out by one of us (S. G.) during the tenure of a U.S. Public Health Service postdoctoral fellowship.

Stantey Garther

Th. DoBZHaNSKY

Institute for the Study of Human Variation, Columbia University,

New York City. June 8.

\section{A Peculiar Nutritional Dermatitis in Pigs}

Is the course of our advisory duties, we have frequently encountered a number of cases of a peculiar dermatitis in pigs. The condition develops in pigs of about 10-12 weeks of age and is characterized initially by the development of red blotches under the skin of the hocks, spreading up the hind legs into the groin and over the belly and eventually over the back and ears. The dermatitis is accompanied by an acute irritation; the pigs become very uncomfortable and rub continually against the sides of the sties. This rubbing usually breaks the skin, resulting in the formation of extensive scabs. Appetite is adversely affected and the pigs make little or no liveweight gain. Natural recovery is very slow, and as a result of the condition, the pigs may take several weeks longer to reach the bacon factory.

The condition develops on both home-mixed and proprietary rations, with or without added antibiotics, and cannot be controlled by normal oily or powder dressings, dipping, bathing or spraying with insecticide preparations. It has only been found when pigs are dry-fed, even though drinking water is freely available.

The dermatitis can be quickly controlled and finally obviated by changing the affected pigs to wet-feeding. The inclusion of fodder beet or potatoes in the diet results in a marked improvement; but the recovery is not so dramatic as that which oceurs when a wet-feeding practice is employed. On wetfeeding, appetite is quickly regained, the scabs slowly disappear and the pigs begin to put on weight again.

There is as yet no evidence of the fundamental cause of the condition or a valid explanation of the mechanism of the empirical cure ; but we are putting these observations on record in the hope that they will be of value to others who may have encountered the condition, which would appear to be of more widespread occurrence than has hitherto been recognized.

Thanks are due to Col. J. E. B. Freeman, Buxhall Vale, Stowmarket, for allowing us facilities for experiments and for his careful observations.

\section{G. THомаS}

A. EDEN

National Agricultural Advisory Service,

Eastern Province, Anstey Hall,

Trumpington, Cambridge. July 31.

\section{Action of Squalene upon Carcinogenic Hydrocarbons}

Squalene is an unsaturated triterpenoid hydrocarbon which was originally isolated from the liver oil of the shark ${ }^{1}$. Among its other occurrences, it was found to be present in the fat from ovarian dermoid cysts $^{2}$. In an investigation on the role of sebum in carcinogenesis, squalene was found to be present in horse smegma and in human ear wax and hair oil ${ }^{3,4}$. MacKenna and co-workers found it in skin surface sebums.

The possible role of squalene as a protective agent in sebum is under investigation in this laboratory. It has already been shown to be fungistatic in vitro against certain dermatophytes ${ }^{6}$. This appears to be related to rapid peroxide formation which occurs when squalene is exposed to air.

Squalene was purified by passage through alumina. 3-Methylcholanthrene, 7,12-dimethylbenz[a]anthra- 